\title{
SOME REVERSES OF THE JENSEN INEQUALITY WITH APPLICATIONS
}

\author{
S. S. DRAGOMIR
}

(Received 23 February 2012; accepted 21 November 2012; first published online 7 February 2013)

\begin{abstract}
Two new reverses of the celebrated Jensen's inequality for convex functions in the general setting of the Lebesgue integral, with applications to means, Hölder's inequality and $f$-divergence measures in information theory, are given.
\end{abstract}

2010 Mathematics subject classification: primary 26D15; secondary 26D20, 94A05.

Keywords and phrases: Jensen's inequality, Hölder's inequality, measurable functions, Lebesgue integral, divergence measures, $f$-divergence measures.

\section{Introduction}

Let $(\Omega, \mathcal{A}, \mu)$ be a measurable space consisting of a set $\Omega$, a $\sigma$-algebra $\mathcal{A}$ of parts of $\Omega$ and a countably additive and positive measure $\mu$ on $\mathcal{A}$ with values in $\mathbb{R} \cup\{\infty\}$. For a $\mu$-measurable function $w: \Omega \rightarrow \mathbb{R}$, with $w(x) \geq 0$ for $\mu$-a.e. (almost every) $x \in \Omega$, consider the Lebesgue space

$$
L_{w}(\Omega, \mu):=\left\{f: \Omega \rightarrow \mathbb{R} \mid f \text { is } \mu \text {-measurable and } \int_{\Omega} w(x)|f(x)| d \mu(x)<\infty\right\} .
$$

For simplicity of notation, we write everywhere in the following $\int_{\Omega} w d \mu$ instead of $\int_{\Omega} w(x) d \mu(x)$.

If $f, g: \Omega \rightarrow \mathbb{R}$ are $\mu$-measurable functions, $\int_{\Omega} w d \mu=1$ and $f, g, f g \in L_{w}(\Omega, \mu)$, then we may consider the $\check{C}$ ebyšev functional

$$
T_{w}(f, g):=\int_{\Omega} w f g d \mu-\int_{\Omega} w f d \mu \int_{\Omega} w g d \mu .
$$

The following result is known in the literature as the Grüss inequality:

$$
\left|T_{w}(f, g)\right| \leq \frac{1}{4}(\Gamma-\gamma)(\Delta-\delta)
$$

(C) 2013 Australian Mathematical Publishing Association Inc. 0004-9727/2013 \$16.00 
provided

$$
-\infty<\gamma \leq f(x) \leq \Gamma<\infty, \quad-\infty<\delta \leq g(x) \leq \Delta<\infty
$$

for $\mu$-a.e. $x \in \Omega$.

The constant $\frac{1}{4}$ is sharp in the sense that it cannot be replaced by a smaller constant.

If we assume that $-\infty<\gamma \leq f(x) \leq \Gamma<\infty$ for $\mu$-a.e. $x \in \Omega$, then, by the Grüss inequality for $g=f$ and by Schwarz's integral inequality,

$$
\int_{\Omega} w\left|f-\int_{\Omega} w f d \mu\right| d \mu \leq\left(\int_{\Omega} w f^{2} d \mu-\left(\int_{\Omega} w f d \mu\right)^{2}\right)^{1 / 2} \leq \frac{1}{2}(\Gamma-\gamma) .
$$

To provide a reverse of the celebrated Jensen's integral inequality for convex functions, in 2002, the author [12] obtained the following result.

Theorem 1.1. Let $\Phi:[m, M] \subset \mathbb{R} \rightarrow \mathbb{R}$ be a differentiable convex function on $(m, M)$ and $f: \Omega \rightarrow[m, M]$ such that $\Phi \circ f, f, \Phi^{\prime} \circ f,\left(\Phi^{\prime} \circ f\right) \cdot f \in L_{w}(\Omega, \mu)$, where $w \geq 0 \mu$ a.e. on $\Omega$ with $\int_{\Omega} w d \mu=1$. Then we have the inequality

$$
\begin{aligned}
0 & \leq \int_{\Omega} w(\Phi \circ f) d \mu-\Phi\left(\int_{\Omega} w f d \mu\right) \\
& \leq \int_{\Omega} w\left(\Phi^{\prime} \circ f\right) f d \mu-\int_{\Omega} w\left(\Phi^{\prime} \circ f\right) d \mu \int_{\Omega} w f d \mu \\
& \leq \frac{1}{2}\left(\Phi^{\prime}(M)-\Phi^{\prime}(m)\right) \int_{\Omega} w\left|f-\int_{\Omega} w f d \mu\right| d \mu .
\end{aligned}
$$

For a generalisation of the first inequality in (1.2) without the differentiability assumption and the derivative $\Phi^{\prime}$ replaced with a selection $\varphi$ from the subdifferential $\partial \Phi$, see Niculescu [27].

If $\mu(\Omega)<\infty$ and $\Phi \circ f, f, \Phi^{\prime} \circ f,\left(\Phi^{\prime} \circ f\right) \cdot f \in L(\Omega, \mu)$, then we have the inequality

$$
\begin{aligned}
0 & \leq \frac{1}{\mu(\Omega)} \int_{\Omega}(\Phi \circ f) d \mu-\Phi\left(\frac{1}{\mu(\Omega)} \int_{\Omega} f d \mu\right) \\
& \leq \frac{1}{\mu(\Omega)} \int_{\Omega}\left(\Phi^{\prime} \circ f\right) f d \mu-\frac{1}{\mu(\Omega)} \int_{\Omega}\left(\Phi^{\prime} \circ f\right) d \mu \cdot \frac{1}{\mu(\Omega)} \int_{\Omega} f d \mu \\
& \leq \frac{1}{2}\left(\Phi^{\prime}(M)-\Phi^{\prime}(m)\right) \frac{1}{\mu(\Omega)} \int_{\Omega}\left|f-\frac{1}{\mu(\Omega)} \int_{\Omega} f d \mu\right| d \mu .
\end{aligned}
$$

The following discrete inequality is of interest as well.

Corollary 1.2. Let $\Phi:[m, M] \rightarrow \mathbb{R}$ be a differentiable convex function on $(m, M)$. If $x_{i} \in[m, M]$ and $w_{i} \geq 0(i=1, \ldots, n)$ with $W_{n}:=\sum_{i=1}^{n} w_{i}=1$, then we have the 
counterpart of Jensen's weighted discrete inequality:

$$
\begin{aligned}
0 & \leq \sum_{i=1}^{n} w_{i} \Phi\left(x_{i}\right)-\Phi\left(\sum_{i=1}^{n} w_{i} x_{i}\right) \\
& \leq \sum_{i=1}^{n} w_{i} \Phi^{\prime}\left(x_{i}\right) x_{i}-\sum_{i=1}^{n} w_{i} \Phi^{\prime}\left(x_{i}\right) \sum_{i=1}^{n} w_{i} x_{i} \\
& \leq \frac{1}{2}\left(\Phi^{\prime}(M)-\Phi^{\prime}(m)\right) \sum_{i=1}^{n} w_{i}\left|x_{i}-\sum_{j=1}^{n} w_{j} x_{j}\right| .
\end{aligned}
$$

REMARK 1.3. The inequality between the first and the second terms in (1.3) was proved in 1994 by Dragomir and Ionescu [15].

Using the results (1.2) and (1.1), we can state the following string of reverse inequalities:

$$
\begin{aligned}
0 & \leq \int_{\Omega} w(\Phi \circ f) d \mu-\Phi\left(\int_{\Omega} w f d \mu\right) \\
& \leq \int_{\Omega} w\left(\Phi^{\prime} \circ f\right) f d \mu-\int_{\Omega} w\left(\Phi^{\prime} \circ f\right) d \mu \int_{\Omega} w f d \mu \\
& \leq \frac{1}{2}\left(\Phi^{\prime}(M)-\Phi^{\prime}(m)\right) \int_{\Omega} w\left|f-\int_{\Omega} w f d \mu\right| d \mu \\
& \leq \frac{1}{2}\left(\Phi^{\prime}(M)-\Phi^{\prime}(m)\right)\left(\int_{\Omega} w f^{2} d \mu-\left(\int_{\Omega} w f d \mu\right)^{2}\right)^{1 / 2} \\
& \leq \frac{1}{4}\left(\Phi^{\prime}(M)-\Phi^{\prime}(m)\right)(M-m),
\end{aligned}
$$

provided that $\Phi:[m, M] \subset \mathbb{R} \rightarrow \mathbb{R}$ is a differentiable convex function on $(m, M)$ and $f:$ $\Omega \rightarrow[m, M]$ such that $\Phi \circ f, f, \Phi^{\prime} \circ f,\left(\Phi^{\prime} \circ f\right) \cdot f \in L_{w}(\Omega, \mu)$, where $w \geq 0 \mu$-a.e. on $\Omega$ with $\int_{\Omega} w d \mu=1$.

Remark 1.4. The inequality between the first, second and last terms from (1.4) was proved in the general case of positive linear functionals in 2001 by the author [11].

Motivated by the above results, we establish in the current paper two new reverses of Jensen's integral inequality for a convex function. Some natural applications for inequalities between means, reverses of Hölder's inequality and for the $f$-divergence measure that play an important role in information theory are given as well.

\section{Reverse inequalities}

The following reverse of Jensen's inequality holds.

Theorem 2.1. Let $\Phi: I \rightarrow \mathbb{R}$ be a continuous convex function on the interval of real

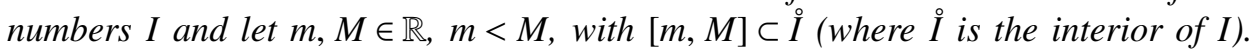


If $f: \Omega \rightarrow \mathbb{R}$ is $\mu$-measurable, satisfies the bounds

$$
-\infty<m \leq f(x) \leq M<\infty \text { for } \mu \text {-a.e. } x \in \Omega
$$

and is such that $f, \Phi \circ f \in L_{w}(\Omega, \mu)$, where $w \geq 0 \mu$-a.e. on $\Omega$ with $\int_{\Omega} w d \mu=1$, then

$$
\begin{aligned}
0 & \leq \int_{\Omega} w(\Phi \circ f) d \mu-\Phi\left(\bar{f}_{\Omega, w}\right) \\
& \leq \frac{\left(M-\bar{f}_{\Omega, w}\right)\left(\bar{f}_{\Omega, w}-m\right)}{M-m} \sup _{t \in(m, M)} \Psi_{\Phi}(t ; m, M) \\
& \leq\left(M-\bar{f}_{\Omega, w}\right)\left(\bar{f}_{\Omega, w}-m\right) \frac{\Phi_{-}^{\prime}(M)-\Phi_{+}^{\prime}(m)}{M-m} \\
& \leq \frac{1}{4}(M-m)\left(\Phi_{-}^{\prime}(M)-\Phi_{+}^{\prime}(m)\right),
\end{aligned}
$$

where $\bar{f}_{\Omega, w}:=\int_{\Omega} w(x) f(x) d \mu(x) \in[m, M]$ and $\Psi_{\Phi}(\cdot ; m, M):(m, M) \rightarrow \mathbb{R}$ is defined by

$$
\Psi_{\Phi}(t ; m, M)=\frac{\Phi(M)-\Phi(t)}{M-t}-\frac{\Phi(t)-\Phi(m)}{t-m} .
$$

We also have the inequality

$$
\begin{aligned}
0 & \leq \int_{\Omega} w(\Phi \circ f) d \mu-\Phi\left(\bar{f}_{\Omega, w}\right) \leq \frac{1}{4}(M-m) \Psi_{\Phi}\left(\bar{f}_{\Omega, w} ; m, M\right) \\
& \leq \frac{1}{4}(M-m)\left(\Phi_{-}^{\prime}(M)-\Phi_{+}^{\prime}(m)\right),
\end{aligned}
$$

provided that $\bar{f}_{\Omega, w} \in(m, M)$.

Proof. By the convexity of $\Phi$,

$$
\begin{aligned}
& \int_{\Omega} w(x) \Phi(f(x)) d \mu(x)-\Phi\left(\bar{f}_{\Omega, w}\right) \\
&=\int_{\Omega} w(x) \Phi\left(\frac{m(M-f(x))+M(f(x)-m)}{M-m}\right) d \mu(x) \\
& \quad-\Phi\left(\int_{\Omega} w(x)\left(\frac{m(M-f(x))+M(f(x)-m)}{M-m}\right) d \mu(x)\right) \\
& \leq \int_{\Omega} \frac{(M-f(x)) \Phi(m)+(f(x)-m) \Phi(M)}{M-m} w(x) d \mu(x) \\
&-\Phi\left(\frac{m\left(M-\bar{f}_{\Omega, w}\right)+M\left(\bar{f}_{\Omega, w}-m\right)}{M-m}\right) \\
&= \frac{\left(M-\bar{f}_{\Omega, w}\right) \Phi(m)+\left(\bar{f}_{\Omega, w}-m\right) \Phi(M)}{M-m} \\
&-\Phi\left(\frac{m\left(M-\bar{f}_{\Omega, w}\right)+M\left(\bar{f}_{\Omega, w}-m\right)}{M-m}\right):=B .
\end{aligned}
$$


By denoting

$$
\Delta_{\Phi}(t ; m, M):=\frac{(t-m) \Phi(M)+(M-t) \Phi(m)}{M-m}-\Phi(t), \quad t \in[m, M],
$$

we have

$$
\begin{aligned}
\Delta_{\Phi}(t ; m, M) & =\frac{(t-m) \Phi(M)+(M-t) \Phi(m)-(M-m) \Phi(t)}{M-m} \\
& =\frac{(t-m) \Phi(M)+(M-t) \Phi(m)-(M-t+t-m) \Phi(t)}{M-m} \\
& =\frac{(t-m)(\Phi(M)-\Phi(t))-(M-t)(\Phi(t)-\Phi(m))}{M-m} \\
& =\frac{(M-t)(t-m)}{M-m} \Psi_{\Phi}(t ; m, M)
\end{aligned}
$$

for any $t \in(m, M)$.

Therefore we have the equality

$$
B=\frac{\left(M-\bar{f}_{\Omega, w}\right)\left(\bar{f}_{\Omega, w}-m\right)}{M-m} \Psi_{\Phi}\left(\bar{f}_{\Omega, w} ; m, M\right),
$$

provided that $\bar{f}_{\Omega, w} \in(m, M)$.

For $\bar{f}_{\Omega, w}=m$ or $\bar{f}_{\Omega, w}=M$ the inequality (2.1) is obvious. If $\bar{f}_{\Omega, w} \in(m, M)$, then

$$
\begin{aligned}
\Psi_{\Phi}\left(\bar{f}_{\Omega, w} ; m, M\right) & \leq \sup _{t \in(m, M)} \Psi_{\Phi}(t ; m, M) \\
& =\sup _{t \in(m, M)}\left(\frac{\Phi(M)-\Phi(t)}{M-t}-\frac{\Phi(t)-\Phi(m)}{t-m}\right) \\
& \leq \sup _{t \in(m, M)}\left(\frac{\Phi(M)-\Phi(t)}{M-t}\right)+\sup _{t \in(m, M)}\left(-\frac{\Phi(t)-\Phi(m)}{t-m}\right) \\
& =\sup _{t \in(m, M)}\left(\frac{\Phi(M)-\Phi(t)}{M-t}\right)-\inf _{t \in(m, M)}\left(\frac{\Phi(t)-\Phi(m)}{t-m}\right) \\
& =\Phi_{-}^{\prime}(M)-\Phi_{+}^{\prime}(m),
\end{aligned}
$$

which by (2.3) and (2.4) produces the desired result (2.1).

Since, obviously,

$$
\frac{\left(M-\bar{f}_{\Omega, w}\right)\left(\bar{f}_{\Omega, w}-m\right)}{M-m} \leq \frac{1}{4}(M-m),
$$

then by (2.3) and (2.4) we deduce the first inequality (2.2). The second part is clear.

Corollary 2.2. Let $\Phi: I \rightarrow \mathbb{R}$ be a continuous convex function on the interval of real numbers $I$ and $m, M \in \mathbb{R}, m<M$, with $[m, M] \subset \stackrel{\circ}{I}$. If $x_{i} \in[m, M]$ and $p_{i} \geq 0$ 
for $i \in\{1, \ldots, n\}$ with $\sum_{i=1}^{n} p_{i}=1$, then we have the inequalities

$$
\begin{aligned}
0 & \leq \sum_{i=1}^{n} p_{i} \Phi\left(x_{i}\right)-\Phi\left(\bar{x}_{p}\right) \\
& \leq \frac{\left(M-\bar{x}_{p}\right)\left(\bar{x}_{p}-m\right)}{M-m} \sup _{t \in(m, M)} \Psi_{\Phi}(t ; m, M) \\
& \leq\left(M-\bar{x}_{p}\right)\left(\bar{x}_{p}-m\right) \frac{\Phi_{-}^{\prime}(M)-\Phi_{+}^{\prime}(m)}{M-m} \\
& \leq \frac{1}{4}(M-m)\left(\Phi_{-}^{\prime}(M)-\Phi_{+}^{\prime}(m)\right),
\end{aligned}
$$

and

$$
\begin{aligned}
0 & \leq \sum_{i=1}^{n} p_{i} \Phi\left(x_{i}\right)-\Phi\left(\bar{x}_{p}\right) \leq \frac{1}{4}(M-m) \Psi_{\Phi}\left(\bar{x}_{p} ; m, M\right) \\
& \leq \frac{1}{4}(M-m)\left(\Phi_{-}^{\prime}(M)-\Phi_{+}^{\prime}(m)\right),
\end{aligned}
$$

where $\bar{x}_{p}:=\sum_{i=1}^{n} p_{i} x_{i} \in(m, M)$.

Remark 2.3. Define the weighted arithmetic mean of the positive $n$-tuple $x=$ $\left(x_{1}, \ldots, x_{n}\right)$ with the nonnegative weights $w=\left(w_{1}, \ldots, w_{n}\right)$ by

$$
A_{n}(w, x):=\frac{1}{W_{n}} \sum_{i=1}^{n} w_{i} x_{i}
$$

where $W_{n}:=\sum_{i=1}^{n} w_{i}>0$, and the weighted geometric mean of the same $n$-tuple by

$$
G_{n}(w, x):=\left(\prod_{i=1}^{n} x_{i}^{w_{i}}\right)^{1 / W_{n}} .
$$

It is well known that the following arithmetic mean-geometric mean inequality holds true:

$$
A_{n}(w, x) \geq G_{n}(w, x)
$$

Applying the inequality between the first and third terms in (2.5) for the convex function $\Phi(t)=-\log t, t>0$,

$$
\begin{aligned}
1 & \leq \frac{A_{n}(w, x)}{G_{n}(w, x)} \leq \exp \left(\frac{1}{M m}\left(M-A_{n}(w, x)\right)\left(A_{n}(w, x)-m\right)\right) \\
& \leq \exp \left(\frac{1}{4} \frac{(M-m)^{2}}{m M}\right)
\end{aligned}
$$

provided that $0<m \leq x_{i} \leq M<\infty$ for $i \in\{1, \ldots, n\}$. 
Also, if we apply the inequality (2.6) for the same function $\Phi$ we obtain

$$
\begin{aligned}
1 & \leq \frac{A_{n}(w, x)}{G_{n}(w, x)} \\
& \leq\left(\left(\frac{M}{A_{n}(w, x)}\right)^{M-A_{n}(w, x)}\left(\frac{m}{A_{n}(w, x)}\right)^{A_{n}(w, x)-m}\right)^{-(M-m) / 4} \\
& \leq \exp \left(\frac{1}{4} \frac{(M-m)^{2}}{m M}\right) .
\end{aligned}
$$

The following result also holds.

THEOREM 2.4. With the assumptions of Theorem 2.1, we have the inequalities

$$
\begin{aligned}
0 & \leq \int_{\Omega} w(\Phi \circ f) d \mu(x)-\Phi\left(\bar{f}_{\Omega, w}\right) \\
& \leq 2 \max \left\{\frac{M-\bar{f}_{\Omega, w}}{M-m}, \frac{\bar{f}_{\Omega, w}-m}{M-m}\right\}\left(\frac{\Phi(m)+\Phi(M)}{2}-\Phi\left(\frac{m+M}{2}\right)\right) \\
& \leq \frac{1}{2} \max \left\{M-\bar{f}_{\Omega, w}, \bar{f}_{\Omega, w}-m\right\}\left(\Phi_{-}^{\prime}(M)-\Phi_{+}^{\prime}(m)\right) .
\end{aligned}
$$

Proof. We first recall the following result obtained by the author in [14] that provides a refinement and a reverse for the weighted Jensen's discrete inequality:

$$
\begin{aligned}
n \min _{i \in\{1, \ldots, n\}}\left\{p_{i}\right\}\left(\frac{1}{n} \sum_{i=1}^{n} \Phi\left(x_{i}\right)-\Phi\left(\frac{1}{n} \sum_{i=1}^{n} x_{i}\right)\right) \\
\quad \leq \frac{1}{P_{n}} \sum_{i=1}^{n} p_{i} \Phi\left(x_{i}\right)-\Phi\left(\frac{1}{P_{n}} \sum_{i=1}^{n} p_{i} x_{i}\right) \\
\quad \leq n \max _{i \in\{1, \ldots, n\}}\left\{p_{i}\right\}\left(\frac{1}{n} \sum_{i=1}^{n} \Phi\left(x_{i}\right)-\Phi\left(\frac{1}{n} \sum_{i=1}^{n} x_{i}\right)\right),
\end{aligned}
$$

where $\Phi: C \rightarrow \mathbb{R}$ is a convex function defined on the convex subset $C$ of the linear space $X,\left\{x_{i}\right\}_{i \in\{1, \ldots, n\}} \subset C$ are vectors and $\left\{p_{i}\right\}_{i \in\{1, \ldots, n\}}$ are nonnegative numbers with $P_{n}:=\sum_{i=1}^{n} p_{i}>0$.

For $n=2$ we deduce from (2.8) that

$$
\begin{aligned}
2 \min & \{t, 1-t\}\left(\frac{\Phi(x)+\Phi(y)}{2}-\Phi\left(\frac{x+y}{2}\right)\right) \\
& \leq t \Phi(x)+(1-t) \Phi(y)-\Phi(t x+(1-t) y) \\
& \leq 2 \max \{t, 1-t\}\left(\frac{\Phi(x)+\Phi(y)}{2}-\Phi\left(\frac{x+y}{2}\right)\right)
\end{aligned}
$$

for any $x, y \in C$ and $t \in[0,1]$. 
If we use the second inequality in (2.9) for the convex function $\Phi: I \rightarrow \mathbb{R}$ and $m, M \in \mathbb{R}, m<M$, with $[m, M] \subset \grave{I}$, we have for $t=\left(M-\bar{f}_{\Omega, w}\right) /(M-m)$ that

$$
\begin{aligned}
& \frac{\left(M-\bar{f}_{\Omega, w}\right) \Phi(m)+\left(\bar{f}_{\Omega, w}-m\right) \Phi(M)}{M-m} \\
& \quad-\Phi\left(\frac{m\left(M-\bar{f}_{\Omega, w}\right)+M\left(\bar{f}_{\Omega, w}-m\right)}{M-m}\right) \\
& \leq 2 \max \left\{\frac{M-\bar{f}_{\Omega, w}}{M-m}, \frac{\bar{f}_{\Omega, w}-m}{M-m}\right\}\left(\frac{\Phi(m)+\Phi(M)}{2}-\Phi\left(\frac{m+M}{2}\right)\right) .
\end{aligned}
$$

Using (2.3) and (2.10) we deduce the first inequality in (2.7).

Since

$$
\frac{\frac{\Phi(m)+\Phi(M)}{2}-\Phi\left(\frac{m+M}{2}\right)}{M-m}=\frac{1}{4}\left(\frac{\Phi(M)-\Phi\left(\frac{m+M}{2}\right)}{M-\frac{m+M}{2}}-\frac{\Phi\left(\frac{m+M}{2}\right)-\Phi(m)}{\frac{m+M}{2}-m}\right)
$$

and, by the gradient inequality,

$$
\frac{\Phi(M)-\Phi\left(\frac{m+M}{2}\right)}{M-\frac{m+M}{2}} \leq \Phi_{-}^{\prime}(M)
$$

and

$$
\frac{\Phi\left(\frac{m+M}{2}\right)-\Phi(m)}{\frac{m+M}{2}-m} \geq \Phi_{+}^{\prime}(m)
$$

then

$$
\frac{\frac{\Phi(m)+\Phi(M)}{2}-\Phi\left(\frac{m+M}{2}\right)}{M-m} \leq \frac{1}{4}\left(\Phi_{-}^{\prime}(M)-\Phi_{+}^{\prime}(m)\right) .
$$

Making use of (2.10) and (2.11), we deduce the last part of (2.7).

COROLlary 2.5. With the assumptions in Corollary 2.2, we have the inequalities

$$
\begin{aligned}
0 & \leq \sum_{i=1}^{n} p_{i} \Phi\left(x_{i}\right)-\Phi\left(\bar{x}_{p}\right) \\
& \leq 2 \max \left\{\frac{M-\bar{x}_{p}}{M-m}, \frac{\bar{x}_{p}-m}{M-m}\right\}\left(\frac{\Phi(m)+\Phi(M)}{2}-\Phi\left(\frac{m+M}{2}\right)\right) \\
& \leq \frac{1}{2} \max \left\{M-\bar{x}_{p}, \bar{x}_{p}-m\right\}\left(\Phi_{-}^{\prime}(M)-\Phi_{+}^{\prime}(m)\right) .
\end{aligned}
$$

ReMARK 2.6. Since, obviously,

$$
\frac{M-\bar{f}_{\Omega, w}}{M-m}, \frac{\bar{f}_{\Omega, w}-m}{M-m} \leq 1,
$$

we obtain from the first inequality in (2.7) the simpler but coarser inequality

$$
0 \leq \int_{\Omega} w(\Phi \circ f) d \mu(x)-\Phi\left(\bar{f}_{\Omega, w}\right) \leq 2\left(\frac{\Phi(m)+\Phi(M)}{2}-\Phi\left(\frac{m+M}{2}\right)\right) .
$$


The discrete version of this result, namely

$$
0 \leq \sum_{i=1}^{n} p_{i} \Phi\left(x_{i}\right)-\Phi\left(\bar{x}_{p}\right) \leq 2\left(\frac{\Phi(m)+\Phi(M)}{2}-\Phi\left(\frac{m+M}{2}\right)\right),
$$

was obtained in 2008 by Simic [34].

REMARK 2.7. With the assumptions in Remark 2.3 we have the following reverse of the arithmetic mean-geometric mean inequality

$$
1 \leq \frac{A_{n}(w, x)}{G_{n}(w, x)} \leq\left(\frac{A(m, M)}{G(m, M)}\right)^{2 \max \left\{\left(M-A_{n}(w, x)\right) /(M-m),\left(A_{n}(w, x)-m\right) /(M-m)\right\}},
$$

where $A(m, M)$ is the arithmetic mean and $G(m, M)$ is the geometric mean of the positive numbers $m$ and $M$.

\section{Applications for the Hölder inequality}

It is well known that if $f \in L_{p}(\Omega, \mu), p>1$, where the Lebesgue space $L_{p}(\Omega, \mu)$ is defined by

$$
L_{p}(\Omega, \mu):=\left\{f: \Omega \rightarrow \mathbb{R} \mid f \text { is } \mu \text {-measurable and } \int_{\Omega}|f(x)|^{p} d \mu(x)<\infty\right\},
$$

and $g \in L_{q}(\Omega, \mu)$ with $1 / p+1 / q=1$ then $f g \in L(\Omega, \mu)=L_{1}(\Omega, \mu)$ and the Hölder inequality holds true:

$$
\int_{\Omega}|f g| d \mu \leq\left(\int_{\Omega}|f|^{p} d \mu\right)^{1 / p}\left(\int_{\Omega}|g|^{p} d \mu\right)^{1 / q}
$$

Assume that $p>1$. If $h: \Omega \rightarrow \mathbb{R}$ is $\mu$-measurable, satisfies the bounds

$$
0<m \leq|h(x)| \leq M<\infty \quad \text { for } \mu \text {-a.e. } x \in \Omega
$$

and is such that $h,|h|^{p} \in L_{w}(\Omega, \mu)$, for a $\mu$-measurable function $w: \Omega \rightarrow \mathbb{R}$, with $w(x) \geq$ 0 for $\mu$-a.e. $x \in \Omega$ and $\int_{\Omega} w d \mu>0$, then, from (2.1),

$$
\begin{aligned}
0 & \leq \frac{\int_{\Omega}|h|^{p} w d \mu}{\int_{\Omega} w d \mu}-\left(\frac{\int_{\Omega}|h| w d \mu}{\int_{\Omega} w d \mu}\right)^{p} \\
& \leq \frac{\left(M-\overline{|h|}_{\Omega, w}\right)\left(\overline{|h|}_{\Omega, w}-m\right)}{M-m} B_{p}(m, M) \\
& \leq p \frac{M^{p-1}-m^{p-1}}{M-m}\left(M-\overline{|h|}_{\Omega, w}\right)\left(\overline{\mid h}_{\Omega, w}-m\right) \\
& \leq \frac{1}{4} p(M-m)\left(M^{p-1}-m^{p-1}\right)
\end{aligned}
$$


where $\bar{h}_{\Omega, w}:=\int_{\Omega}|h| w d \mu / \int_{\Omega} w d \mu \in[m, M], \Psi_{p}(\cdot ; m, M):(m, M) \rightarrow \mathbb{R}$ is defined by

$$
\Psi_{p}(t ; m, M)=\frac{M^{p}-t^{p}}{M-t}-\frac{t^{p}-m^{p}}{t-m}
$$

and

$$
B_{p}(m, M):=\sup _{t \in(m, M)} \Psi_{p}(t ; m, M)
$$

From (2.2) we also have the inequality

$$
\begin{aligned}
0 & \leq \frac{\int_{\Omega}|h|^{p} w d \mu}{\int_{\Omega} w d \mu}-\left(\frac{\int_{\Omega}|h| w d \mu}{\int_{\Omega} w d \mu}\right)^{p} \leq \frac{1}{4}(M-m) \Psi_{p}\left(|\overline{\mid h}|_{\Omega, w} ; m, M\right) \\
& \leq \frac{1}{4} p(M-m)\left(M^{p-1}-m^{p-1}\right) .
\end{aligned}
$$

Proposition 3.1. If $f \in L_{p}(\Omega, \mu), g \in L_{q}(\Omega, \mu)$ with $p>1,1 / p+1 / q=1$, and there exist constants $\gamma, \Gamma>0$ such that

$$
\gamma \leq \frac{|f|}{|g|^{q-1}} \leq \Gamma \mu \text {-a.e on } \Omega,
$$

then

$$
\begin{aligned}
0 & \leq \frac{\int_{\Omega}|f|^{p} d \mu}{\int_{\Omega}|g|^{q} d \mu}-\left(\frac{\int_{\Omega}|f g| d \mu}{\int_{\Omega}|g|^{q} d \mu}\right)^{p} \\
& \leq \frac{B_{p}(\gamma, \Gamma)}{\Gamma-\gamma}\left(\Gamma-\frac{\int_{\Omega}|f g| d \mu}{\int_{\Omega}|g|^{q} d \mu}\right)\left(\frac{\int_{\Omega}|f g| d \mu}{\int_{\Omega}|g|^{q} d \mu}-\gamma\right) \\
& \leq p \frac{\Gamma^{p-1}-\gamma^{p-1}}{\Gamma-\gamma}\left(\Gamma-\frac{\int_{\Omega}|f g| d \mu}{\int_{\Omega}|g|^{q} d \mu}\right)\left(\frac{\int_{\Omega}|f g| d \mu}{\int_{\Omega}|g|^{q} d \mu}-\gamma\right) \\
& \leq \frac{1}{4} p(\Gamma-\gamma)\left(\Gamma^{p-1}-\gamma^{p-1}\right),
\end{aligned}
$$

and

$$
\begin{aligned}
0 & \leq \frac{\int_{\Omega}|f|^{p} d \mu}{\int_{\Omega}|g|^{q} d \mu}-\left(\frac{\int_{\Omega}|f g| d \mu}{\int_{\Omega}|g|^{q} d \mu}\right)^{p} \\
& \leq \frac{1}{4}(\Gamma-\gamma) \Psi_{p}\left(\frac{\int_{\Omega}|f g| d \mu}{\int_{\Omega}|g|^{q} d \mu} ; \gamma, \Gamma\right) \leq \frac{1}{4} p(\Gamma-\gamma)\left(\Gamma^{p-1}-\gamma^{p-1}\right),
\end{aligned}
$$

where $B_{p}(\cdot, \cdot)$ and $\Psi_{p}(\cdot ; \cdot, \cdot)$ are defined above.

Proof. The inequalities (3.3) and (3.4) follow from (3.1) and (3.2) by choosing

$$
h=\frac{|f|}{|g|^{q-1}} \quad \text { and } \quad w=|g|^{q} .
$$

The details are omitted. 
REMARK 3.2. We observe that for $p=q=2$ we have $\Psi_{2}(t ; \gamma, \Gamma)=\Gamma-\gamma=B_{2}(\gamma, \Gamma)$ and then from the first inequality in (3.3) we get the following reverse of the CauchyBunyakovsky-Schwarz inequality:

$$
\begin{aligned}
& \int_{\Omega}|g|^{2} d \mu \int_{\Omega}|f|^{2} d \mu-\left(\int_{\Omega}|f g| d \mu\right)^{2} \\
& \quad \leq\left(\Gamma-\frac{\int_{\Omega}|f g| d \mu}{\int_{\Omega}|g|^{2} d \mu}\right)\left(\frac{\int_{\Omega}|f g| d \mu}{\int_{\Omega}|g|^{2} d \mu}-\gamma\right)\left(\int_{\Omega}|g|^{2} d \mu\right)^{2},
\end{aligned}
$$

provided that $f, g \in L_{2}(\Omega, \mu)$, and there exist constants $\gamma, \Gamma>0$ such that

$$
\gamma \leq \frac{|f|}{|g|} \leq \Gamma \mu \text {-a.e on } \Omega .
$$

Corollary 3.3. With the assumptions of Proposition 3.1 we have the following additive reverses of the Hölder inequality:

$$
\begin{aligned}
0 \leq & \left(\int_{\Omega}|f|^{p} d \mu\right)^{1 / p}\left(\int_{\Omega}|g|^{q} d \mu\right)^{1 / q}-\int_{\Omega}|f g| d \mu \\
\leq & \left(\frac{B_{p}(\gamma, \Gamma)}{\Gamma-\gamma}\right)^{1 / p}\left(\Gamma-\frac{\int_{\Omega}|f g| d \mu}{\int_{\Omega}|g|^{q} d \mu}\right)^{1 / p}\left(\frac{\int_{\Omega}|f g| d \mu}{\int_{\Omega}|g|^{q} d \mu}-\gamma\right)^{1 / p} \int_{\Omega}|g|^{q} d \mu \\
\leq & p^{1 / p}\left(\frac{\Gamma^{p-1}-\gamma^{p-1}}{\Gamma-\gamma}\right)^{1 / p}\left(\Gamma-\frac{\int_{\Omega}|f g| d \mu}{\int_{\Omega}|g|^{q} d \mu}\right)^{1 / p}\left(\frac{\int_{\Omega}|f g| d \mu}{\int_{\Omega}|g|^{q} d \mu}-\gamma\right)^{1 / p} \\
& \times \int_{\Omega}|g|^{q} d \mu \\
\leq & \frac{1}{4^{1 / p}} p^{1 / p}(\Gamma-\gamma)^{1 / p}\left(\Gamma^{p-1}-\gamma^{p-1}\right)^{1 / p} \int_{\Omega}|g|^{q} d \mu
\end{aligned}
$$

and

$$
\begin{aligned}
0 & \leq\left(\int_{\Omega}|f|^{p} d \mu\right)^{1 / p}\left(\int_{\Omega}|g|^{q} d \mu\right)^{1 / q}-\int_{\Omega}|f g| d \mu \\
& \leq \frac{1}{4^{1 / p}}(\Gamma-\gamma)^{1 / p} \Psi_{p}^{1 / p}\left(\frac{\int_{\Omega}|f g| d \mu}{\int_{\Omega}|g|^{q} d \mu} ; m, M\right) \int_{\Omega}|g|^{q} d \mu \\
& \leq \frac{1}{4^{1 / p}} p^{1 / p}(\Gamma-\gamma)^{1 / p}\left(\Gamma^{p-1}-\gamma^{p-1}\right)^{1 / p} \int_{\Omega}|g|^{q} d \mu,
\end{aligned}
$$

where $p>1$ and $1 / p+1 / q=1$. 
Proof. By multiplying in (3.3) with $\left(\int_{\Omega}|g|^{q} d \mu\right)^{p}$,

$$
\begin{aligned}
\int_{\Omega}|f|^{p} d \mu\left(\int_{\Omega}|g|^{q} d \mu\right)^{p-1}-\left(\int_{\Omega}|f g| d \mu\right)^{p} \\
\quad \leq \frac{B_{p}(\gamma, \Gamma)}{\Gamma-\gamma}\left(\Gamma-\frac{\int_{\Omega}|f g| d \mu}{\int_{\Omega}|g|^{q} d \mu}\right)\left(\frac{\int_{\Omega}|f g| d \mu}{\int_{\Omega}|g|^{q} d \mu}-\gamma\right)\left(\int_{\Omega}|g|^{q} d \mu\right)^{p} \\
\quad \leq p \frac{\Gamma^{p-1}-\gamma^{p-1}}{\Gamma-\gamma}\left(\Gamma-\frac{\int_{\Omega}|f g| d \mu}{\int_{\Omega}|g|^{q} d \mu}\right)\left(\frac{\int_{\Omega}|f g| d \mu}{\int_{\Omega}|g|^{q} d \mu}-\gamma\right)\left(\int_{\Omega}|g|^{q} d \mu\right)^{p} \\
\quad \leq \frac{1}{4} p(\Gamma-\gamma)\left(\Gamma^{p-1}-\gamma^{p-1}\right)\left(\int_{\Omega}|g|^{q} d \mu\right)^{p},
\end{aligned}
$$

which is equivalent to

$$
\begin{array}{rl}
\int_{\Omega}|f|^{p} & d \mu\left(\int_{\Omega}|g|^{q} d \mu\right)^{p-1} \\
\leq & \left(\int_{\Omega}|f g| d \mu\right)^{p}+\frac{B_{p}(\gamma, \Gamma)}{\Gamma-\gamma}\left(\Gamma-\frac{\int_{\Omega}|f g| d \mu}{\int_{\Omega}|g|^{q} d \mu}\right)\left(\frac{\int_{\Omega}|f g| d \mu}{\int_{\Omega}|g|^{q} d \mu}-\gamma\right) \\
& \times\left(\int_{\Omega}|g|^{q} d \mu\right)^{p} \\
\leq & \left(\int_{\Omega}|f g| d \mu\right)^{p}+p\left(\Gamma-\frac{\int_{\Omega}|f g| d \mu}{\int_{\Omega}|g|^{q} d \mu}\right)\left(\frac{\int_{\Omega}|f g| d \mu}{\int_{\Omega}|g|^{q} d \mu}-\gamma\right) \\
& \times\left(\int_{\Omega}|g|^{q} d \mu\right)^{p} \frac{\Gamma^{p-1}-\gamma^{p-1}}{\Gamma-\gamma} \\
\leq & \left(\int_{\Omega}|f g| d \mu\right)^{p}+\frac{1}{4} p(\Gamma-\gamma)\left(\Gamma^{p-1}-\gamma^{p-1}\right)\left(\int_{\Omega}|g|^{q} d \mu\right)^{p} .
\end{array}
$$

Raising to the power $1 / p$ with $p>1$ and employing the elementary inequality that for $p>1$ and $\alpha, \beta>0$,

$$
(\alpha+\beta)^{1 / p} \leq \alpha^{1 / p}+\beta^{1 / p}
$$

we have from the first part of (3.7) that

$$
\begin{aligned}
\left(\int_{\Omega}|f|^{p} d \mu\right)^{1 / p}\left(\int_{\Omega}|g|^{q} d \mu\right)^{1-1 / p} & \\
\leq & \int_{\Omega}|f g| d \mu+\left(\frac{B_{p}(\gamma, \Gamma)}{\Gamma-\gamma}\right)^{1 / p}\left(\Gamma-\frac{\int_{\Omega}|f g| d \mu}{\int_{\Omega}|g|^{q} d \mu}\right)^{1 / p}\left(\frac{\int_{\Omega}|f g| d \mu}{\int_{\Omega}|g|^{q} d \mu}-\gamma\right)^{1 / p} \\
& \times \int_{\Omega}|g|^{q} d \mu
\end{aligned}
$$

and since $1-1 / p=1 / q$ we get from (3.8) the first inequality in (3.5). The rest is obvious.

The inequality (3.6) can be proved in a similar manner; the details are omitted. 
If $h: \Omega \rightarrow \mathbb{R}$ is $\mu$-measurable, satisfies the bounds

$$
0<m \leq|h(x)| \leq M<\infty \quad \text { for } \mu \text {-a.e. } x \in \Omega
$$

and is such that $h,|h|^{p} \in L_{w}(\Omega, \mu)$, for a $\mu$-measurable function $w: \Omega \rightarrow \mathbb{R}$, with $w(x) \geq$ 0 for $\mu$-a.e. $x \in \Omega$ and $\int_{\Omega} w d \mu>0$, then from (2.7) we also have the inequality

$$
\begin{aligned}
0 & \leq \frac{\int_{\Omega}|h|^{p} w d \mu}{\int_{\Omega} w d \mu}-\left(\frac{\int_{\Omega}|h| w d \mu}{\int_{\Omega} w d \mu}\right)^{p} \\
& \leq 2\left(\frac{m^{p}+M^{p}}{2}-\left(\frac{m+M}{2}\right)^{p}\right) \max \left\{\frac{M-\overline{\mid h}_{\Omega, w}}{M-m}, \frac{\overline{\mid h}_{\Omega, w}-m}{M-m}\right\} \\
& \leq \frac{1}{2} p\left(M^{p-1}-m^{p-1}\right) \max \left\{M-\overline{|h|}_{\Omega, w}, \overline{|h|}_{\Omega, w}-m\right\}
\end{aligned}
$$

where, as above, $\overline{|h|}_{\Omega, w}:=\int_{\Omega}|h| w d \mu / \int_{\Omega} w d \mu \in[m, M]$.

From (3.9) we can state the following result.

Proposition 3.4. With the assumptions of Proposition 3.1 we have

$$
\begin{aligned}
0 & \leq \frac{\int_{\Omega}|f|^{p} d \mu}{\int_{\Omega}|g|^{q} d \mu}-\left(\frac{\int_{\Omega}|f g| d \mu}{\int_{\Omega}|g|^{q} d \mu}\right)^{p} \\
& \leq 2 \cdot \frac{\frac{\gamma^{p}+\Gamma^{p}}{2}-\left(\frac{\gamma+\Gamma}{2}\right)^{p}}{\Gamma-\gamma} \max \left\{\Gamma-\frac{\int_{\Omega}|f g| d \mu}{\int_{\Omega}|g|^{q} d \mu}, \frac{\int_{\Omega}|f g| d \mu}{\int_{\Omega}|g|^{q} d \mu}-\gamma\right\} \\
& \leq \frac{1}{2} p\left(\Gamma^{p-1}-\gamma^{p-1}\right) \max \left\{\Gamma-\frac{\int_{\Omega}|f g| d \mu}{\int_{\Omega}|g|^{q} d \mu}, \frac{\int_{\Omega}|f g| d \mu}{\int_{\Omega}|g|^{q} d \mu}-\gamma\right\}
\end{aligned}
$$

Finally, the following additive reverse of the Hölder inequality can also be stated.

Corollary 3.5. With the assumptions of Proposition 3.1,

$$
\begin{aligned}
0 \leq & \left(\int_{\Omega}|f|^{p} d \mu\right)^{1 / p}\left(\int_{\Omega}|g|^{q} d \mu\right)^{1 / q}-\int_{\Omega}|f g| d \mu \\
\leq & 2^{1 / p} \cdot\left(\frac{\frac{\gamma^{p}+\Gamma^{p}}{2}-\left(\frac{\gamma+\Gamma}{2}\right)^{p}}{\Gamma-\gamma}\right)^{1 / p} \\
& \times \max \left\{\left(\Gamma-\frac{\int_{\Omega}|f g| d \mu}{\int_{\Omega}|g|^{q} d \mu}\right)^{1 / p},\left(\frac{\int_{\Omega}|f g| d \mu}{\int_{\Omega}|g|^{q} d \mu}-\gamma\right)^{1 / p}\right\} \int_{\Omega}|g|^{q} d \mu \\
\leq & \frac{1}{2^{1 / p}} p^{1 / p} \max \left\{\left(\Gamma-\frac{\int_{\Omega}|f g| d \mu}{\int_{\Omega}|g|^{q} d \mu}\right)^{1 / p},\left(\frac{\int_{\Omega}|f g| d \mu}{\int_{\Omega}|g|^{q} d \mu}-\gamma\right)^{1 / p}\right\} \\
& \times\left(\Gamma^{p-1}-\gamma^{p-1}\right)^{1 / p} \int_{\Omega}|g|^{q} d \mu .
\end{aligned}
$$


REMARK 3.6. As a simpler but coarser inequality we have the following result:

$$
\begin{aligned}
0 & \leq\left(\int_{\Omega}|f|^{p} d \mu\right)^{1 / p}\left(\int_{\Omega}|g|^{q} d \mu\right)^{1 / q}-\int_{\Omega}|f g| d \mu \\
& \leq 2^{1 / p} \cdot\left(\frac{\gamma^{p}+\Gamma^{p}}{2}-\left(\frac{\gamma+\Gamma}{2}\right)^{p}\right)^{1 / p} \int_{\Omega}|g|^{q} d \mu,
\end{aligned}
$$

where $f$ and $g$ are as above.

\section{Applications for $f$-divergence}

One of the important issues in many applications of probability theory is finding an appropriate measure of distance (or difference or discrimination) between two probability distributions. A number of divergence measures for this purpose have been proposed and extensively studied by Jeffreys [19], Kullback and Leibler [24], Rényi [30], Havrda and Charvat [17], Kapur [22], Sharma and Mittal [32], Burbea and Rao [4], Rao [29], Lin [25], Csiszár [7], Ali and Silvey [1], Vajda [39], Shioya and Da-Te [33] and others (see, for example, [26], and the references therein).

These measures have been applied in a variety of fields such as: anthropology [29], genetics [26], finance, economics and political science [31, 36, 37], biology [28], the analysis of contingency tables [16], approximation of probability distributions [6, 23], signal processing [20, 21] and pattern recognition [2, 5]. A number of these measures of distance are specific cases of Csiszár $f$-divergence and so further exploration of this concept will have a flow-on effect to other measures of distance and to areas in which they are applied.

Assume that a set $\Omega$ and the $\sigma$-finite measure $\mu$ are given. Consider the set of all probability densities on $\mu$ to be $\mathcal{P}:=\left\{p \mid p: \Omega \rightarrow \mathbb{R}, p(x) \geq 0, \int_{\Omega} p(x) d \mu(x)=1\right\}$. The Kullback-Leibler divergence [24] is well known among the information divergences. It is defined as

$$
D_{K L}(p, q):=\int_{\Omega} p(x) \log \left(\frac{p(x)}{q(x)}\right) d \mu(x), \quad p, q \in \mathcal{P},
$$

where $\log$ is to base $e$.

In information theory and statistics, various divergences are applied in addition to the Kullback-Leibler divergence. These are, for example, the variation distance $D_{v}$, Hellinger distance $D_{H}[18], \chi^{2}$-divergence $D_{\chi^{2}}, \alpha$-divergence $D_{\alpha}$, Bhattacharyya distance $D_{B}[3]$, harmonic distance $D_{H a}$, Jeffreys distance $D_{J}[19]$, triangular discrimination $D_{\Delta}[38]$. They are defined as follows:

$$
\begin{aligned}
D_{v}(p, q) & :=\int_{\Omega}|p(x)-q(x)| d \mu(x), \quad p, q \in \mathcal{P} \\
D_{H}(p, q) & :=\int_{\Omega}|\sqrt{p(x)}-\sqrt{q(x)}| d \mu(x), \quad p, q \in \mathcal{P}
\end{aligned}
$$




$$
\begin{gathered}
D_{\chi^{2}}(p, q):=\int_{\Omega} p(x)\left(\left(\frac{q(x)}{p(x)}\right)^{2}-1\right) d \mu(x), \quad p, q \in \mathcal{P} \\
D_{\alpha}(p, q):=\frac{4}{1-\alpha^{2}}\left(1-\int_{\Omega}(p(x))^{(1-\alpha) / 2}(q(x))^{(1+\alpha) / 2} d \mu(x)\right), \quad p, q \in \mathcal{P} ; \\
D_{B}(p, q):=\int_{\Omega} \sqrt{p(x) q(x)} d \mu(x), \quad p, q \in \mathcal{P} ; \\
D_{H a}(p, q):=\int_{\Omega} \frac{2 p(x) q(x)}{p(x)+q(x)} d \mu(x), \quad p, q \in \mathcal{P} \\
D_{J}(p, q):=\int_{\Omega}(p(x)-q(x)) \log \left(\frac{p(x)}{q(x)}\right) d \mu(x), \quad p, q \in \mathcal{P} \\
D_{\Delta}(p, q):=\int_{\Omega} \frac{(p(x)-q(x))^{2}}{p(x)+q(x)} d \mu(x), \quad p, q \in \mathcal{P} .
\end{gathered}
$$

For other divergence measures, see Kapur [22] or the book online by Taneja [35].

Csiszár $f$-divergence is defined as follows [8]:

$$
I_{f}(p, q):=\int_{\Omega} p(x) f\left(\frac{q(x)}{p(x)}\right) d \mu(x), \quad p, q \in \mathcal{P},
$$

where $f$ is convex on $(0, \infty)$. It is assumed that $f$ is strictly convex and satisfies the condition that $f(1)=0$. By appropriately defining this convex function, various divergences are derived. Most of the above distances (4.1)-(4.9) are particular instances of Csiszár $f$-divergence. There are also many others which are not in this class (see, for example, [35]). For the basic properties of Csiszár $f$-divergence, see [8, 9] and [39].

The following result holds.

Proposition 4.1. Suppose that $f:(0, \infty) \rightarrow \mathbb{R}$ be a convex function with the property that $f(1)=0$. Assume that $p, q \in \mathcal{P}$ and there exist constants $0<r<1<R<\infty$ such that

$$
r \leq \frac{q(x)}{p(x)} \leq R \quad \text { for } \mu \text {-a.e. } x \in \Omega .
$$

Then we have the inequalities

$$
\begin{aligned}
I_{f}(p, q) & \leq \frac{(R-1)(1-r)}{R-r} \sup _{t \in(r, R)} \Psi_{f}(t ; r, R) \\
& \leq(R-1)(1-r) \frac{f_{-}^{\prime}(R)-f_{+}^{\prime}(r)}{R-r} \\
& \leq \frac{1}{4}(R-r)\left(f_{-}^{\prime}(R)-f_{+}^{\prime}(r)\right),
\end{aligned}
$$

where $\Psi_{f}(\cdot ; r, R):(r, R) \rightarrow \mathbb{R}$ is defined by

$$
\Psi_{f}(t ; r, R)=\frac{f(R)-f(t)}{R-t}-\frac{f(t)-f(r)}{t-r} .
$$


We also have the inequality

$$
\begin{aligned}
I_{f}(p, q) & \leq \frac{1}{4}(R-r) \frac{f(R)(1-r)+f(r)(R-1)}{(R-1)(1-r)} \\
& \leq \frac{1}{4}(R-r)\left(f_{-}^{\prime}(R)-f_{+}^{\prime}(r)\right) .
\end{aligned}
$$

The proof follows by Theorem 2.1 by choosing $w(x)=p(x), f(x)=q(x) / p(x), m=r$ and $M=R$ and performing the required calculations. The details are omitted.

Using the same approach and Theorem 2.4 we can also state the following result.

Proposition 4.2. With the assumptions of Proposition 4.1,

$$
\begin{aligned}
I_{f}(p, q) & \leq 2 \max \left\{\frac{R-1}{R-r}, \frac{1-r}{R-r}\right\}\left(\frac{f(r)+f(R)}{2}-f\left(\frac{r+R}{2}\right)\right) \\
& \leq \frac{1}{2} \max \{R-1,1-r\}\left(f_{-}^{\prime}(R)-f_{+}^{\prime}(r)\right) .
\end{aligned}
$$

The above results can be used to obtain various inequalities for divergence measures in information theory that are particular instances of $f$-divergence.

Consider the Kullback-Leibler divergence

$$
D_{K L}(p, q):=\int_{\Omega} p(x) \log \left(\frac{p(x)}{q(x)}\right) d \mu(x), \quad p, q \in \mathcal{P},
$$

which is an $f$-divergence for the convex function $f:(0, \infty) \rightarrow \mathbb{R}, f(t)=-\log t$.

If $p, q \in \mathcal{P}$ such that there exist constants $0<r<1<R<\infty$ with

$$
r \leq \frac{q(x)}{p(x)} \leq R \quad \text { for } \mu \text {-a.e. } x \in \Omega,
$$

then we get from (4.10) that

$$
D_{K L}(p, q) \leq \frac{(R-1)(1-r)}{r R},
$$

from (4.11) that

$$
D_{K L}(p, q) \leq \frac{1}{4}(R-r) \log \left(R^{-1 /(R-1)} r^{-1 /(1-r)}\right)
$$

and from (4.12) that

$$
\begin{aligned}
D_{K L}(p, q) & \leq 2 \max \left\{\frac{R-1}{R-r}, \frac{1-r}{R-r}\right\} \log \left(\frac{A(r, R)}{G(r, R)}\right) \\
& \leq \frac{1}{2} \max \{R-1,1-r\}\left(\frac{R-r}{r R}\right),
\end{aligned}
$$

where $A(r, R)$ is the arithmetic mean and $G(r, R)$ is the geometric mean of the positive numbers $r$ and $R$. 


\section{Acknowledgement}

The author would like to thank the anonymous referee for valuable remarks that have been implemented in the final version of the paper.

\section{References}

[1] S. M. Ali and S. D. Silvey, 'A general class of coefficients of divergence of one distribution from another', J. R. Stat. Soc. Ser. B 28 (1966), 131-142.

[2] M. Ben-Bassat, ' $f$-entropies, probability of error and feature selection', Inform. Control 39 (1978), $227-242$.

[3] A. Bhattacharyya, 'On a measure of divergence between two statistical populations defined by their probability distributions', Bull. Calcutta Math. Soc. 35 (1943), 99-109.

[4] I. Burbea and C. R. Rao, 'On the convexity of some divergence measures based on entropy function', IEEE Trans. Inform. Theory 28(3) (1982), 489-495.

[5] C. H. Chen, Statistical Pattern Recognition (Hoyderc Book Co., Rocelle Park, New York, 1973).

[6] C. K. Chow and C. N. Lin, 'Approximating discrete probability distributions with dependence trees', IEEE Trans. Inform. Theory 14(3) (1968), 462-467.

[7] I. Csiszár, 'Information-type measures of difference of probability distributions and indirect observations', Studia Math. Hungarica 2 (1967), 299-318.

[8] I. Csiszár, 'On topological properties of $f$-divergences', Studia Math. Hungarica 2 (1967), 329339.

[9] I. Csiszár and J. Körner, Information Theory: Coding Theorem for Discrete Memoryless Systems (Academic Press, New York, 1981).

[10] S. S. Dragomir, 'A converse result for Jensen's discrete inequality via Gruss' inequality and applications in information theory', An. Univ. Oradea Fasc. Mat. 7 (1999/2000), 178-189.

[11] S. S. Dragomir, 'On a reverse of Jensen's inequality for isotonic linear functionals', J. Inequal. Pure Appl. Math. 2(3) (2001), (Article 36).

[12] S. S. Dragomir, 'A Grüss type inequality for isotonic linear functionals and applications', Demonstratio Math. 36(3) (2003), 551-562.

[13] S. S. Dragomir, 'Bounds for the deviation of a function from the chord generated by its extremities', Bull. Aust. Math. Soc. 78(2) (2008), 225-248.

[14] S. S. Dragomir, 'Bounds for the normalized Jensen functional', Bull. Aust. Math. Soc. 74(3) (2006), 471-476.

[15] S. S. Dragomir and N. M. Ionescu, 'Some converse of Jensen's inequality and applications', Rev. Anal. Numér. Théor. Approx. 23(1) (1994), 71-78.

[16] D. V. Gokhale and S. Kullback, Information in Contingency Tables (Marcel Decker, New York, 1978).

[17] J. H. Havrda and F. Charvat, 'Quantification method classification process: concept of structural $\alpha$-entropy', Kybernetika 3 (1967), 30-35.

[18] E. Hellinger, 'Neue Begründung der Theorie quadratischer Formen von unendlichvielen Veränderlichen', J. reine angew. Math. 36 (1909), 210-271.

[19] H. Jeffreys, 'An invariant form for the prior probability in estimating problems', Proc. R. Soc. Lond. A 186 (1946), 453-461.

[20] T. T. Kadota and L. A. Shepp, 'On the best finite set of linear observables for discriminating two Gaussian signals', IEEE Trans. Inform. Theory 13 (1967), 288-294.

[21] T. Kailath, 'The divergence and Bhattacharyya distance measures in signal selection', IEEE Trans. Comm. Technology. (1967), 52-60; (Vol COM-15).

[22] J. N. Kapur, 'A comparative assessment of various measures of directed divergence', Adv. Management Stud. 3 (1984), 1-16.

[23] D. Kazakos and T. Cotsidas, "A decision theory approach to the approximation of discrete probability densities', IEEE Trans. Perform. Anal. Machine Intell. 1 (1980), 61-67. 
[24] S. Kullback and R. A. Leibler, 'On information and sufficiency', Annals Math. Statist. 22 (1951), 79-86.

[25] J. Lin, 'Divergence measures based on the Shannon entropy', IEEE Trans. Inform. Theory 37(1) (1991), 145-151.

[26] M. Mei, 'The theory of genetic distance and evaluation of human races', Japan J. Human Genetics 23 (1978), 341-369.

[27] C. P. Niculescu, 'An extension of Chebyshev's inequality and its connection with Jensen's inequality', J. Inequal. Appl. 6(4) (2001), 451-462.

[28] E. C. Pielou, Ecological Diversity (Wiley, New York, 1975).

[29] C. R. Rao, 'Diversity and dissimilarity coefficients: a unified approach', Theoretic Population Biol. 21 (1982), 24-43.

[30] A. Rényi, 'On measures of entropy and information', Proc. Fourth Berkeley Symp. Math. Stat. Prob. 1 (1961), 547-561, (University of California Press).

[31] A. Sen, On Economic Inequality (Oxford University Press, London, 1973).

[32] B. D. Sharma and D. P. Mittal, 'New nonadditive measures of relative information', J. Comb. Inf. Syst. Sci. 2(4) (1977), 122-132.

[33] H. Shioya and T. Da-Te, 'A generalisation of Lin divergence and the derivative of a new information divergence', Elec. and Comm. in Japan 78(7) (1995), 37-40.

[34] S. Simic, 'On a global upper bound for Jensen's inequality', J. Math. Anal. Appl. 343 (2008), 414-419.

[35] I. J. Taneja, Generalised Information Measures and their Applications, http://www.mtm.ufsc.br/-taneja/bhtml/bhtml.html.

[36] H. Theil, Economics and Information Theory (North-Holland, Amsterdam, 1967).

[37] H. Theil, Statistical Decomposition Analysis (North-Holland, Amsterdam, 1972).

[38] F. Topsoe, 'Some inequalities for information divergence and related measures of discrimination', Res. Rep. Coll., RGMIA 2(1) (1999), 85-98.

[39] I. Vajda, Theory of Statistical Inference and Information (Kluwer Academic Publishers, Dordrecht-Boston, 1989).

S. S. DRAGOMIR, Mathematics, School of Engineering \& Science, Victoria University, PO Box 14428, Melbourne City, MC 8001, Australia

and

School of Computational \& Applied Mathematics, University of the Witwatersrand, Private Bag 3,

Johannesburg 2050, South Africa

e-mail: sever.dragomir@vu.edu.au 